PROCEEDINGS OF THE

AMERICAN MATHEMATICAL SOCIETY

Volume 137, Number 11, November 2009, Pages 3923-3926

S 0002-9939(09)09958-4

Article electronically published on June 12, 2009

\title{
VANISHING OF EXTENSIONS OF TWISTED VERMA MODULES
}

\author{
NORIYUKI ABE
}

(Communicated by Gail R. Letzter)

\begin{abstract}
We prove a vanishing theorem of extension groups between twisted Verma modules.
\end{abstract}

\section{INTRODUCTION}

The notion of twisted (or shuffled) Verma modules was introduced by Irving Irv93 and also developed by Andersen and Lauritzen AL03. These are objects of the Bernstein-Gelfand-Gelfand category $\mathcal{O}$ [BGG76] which correspond to the principal series representations by the Bernstein-Gelfand equivalence of categories BG80].

In this paper, we prove a vanishing theorem of extension groups between twisted Verma modules. In the case of Verma modules, this theorem was proved by Schmid [Sch81 and Delorme Del80. (See also Car86, Proposition 3.7].)

Let $\mathfrak{g}$ be a complex semisimple Lie algebra. Fix a Cartan subalgebra $\mathfrak{h}$ and a Borel subalgebra $\mathfrak{b}$. These data define the Bernstein-Gelfand-Gelfand category $\mathcal{O}$ [BGG76, Definition 1]. Let $\rho$ be the half-sum of positive roots, $W$ the Weyl group and $w_{0}$ its longest element. We denote $x<y$ for $x, y \in W$ if $x$ is less than $y$ with respect to the Bruhat order. For $w \in W$, let $\ell(w)$ be the length of $w$. For $x \in W$, we denote the Verma module with highest weight $x \rho-\rho$ by $M(x)$. Let $T_{w}$ be a twisting functor for $w \in W$ AL03, 6.1].

To state our main result, we define a subset of the Weyl group $A_{w}(x)$ indexed by $x, w \in W$. Let $w=s_{1} \cdots s_{l}$ be a reduced expression and set $t_{i}=$ $\left(s_{1} \cdots s_{i-1}\right) s_{i}\left(s_{1} \cdots s_{i-1}\right)^{-1}$. Put

$$
A_{w}(x)=\left\{\begin{array}{l|l}
z \in W & \begin{array}{l}
\text { for some } 1 \leq i_{1}<\cdots<i_{r} \leq l, z=t_{i_{r}} \cdots t_{i_{1}} x \text { and } \\
t_{i_{k}} t_{i_{k-1}} \cdots t_{i_{1}} x<t_{i_{k-1}} \cdots t_{i_{1}} x \text { for all } k=1, \ldots, r
\end{array}
\end{array}\right\} .
$$

Then by [Abe, this set is independent of the choice of a reduced expression. Put

$$
\ell(w, x, v, y)=\max \left\{2 \ell(z)-\ell(w x)-\ell(v y) \mid z \in w A_{w^{-1}}(x) \cap v w_{0} A_{w_{0} v^{-1}}\left(w_{0} y\right)\right\} .
$$

(If $w A_{w^{-1}}(x) \cap v w_{0} A_{w_{0} v^{-1}}\left(w_{0} y\right)=\emptyset$, put $\ell(w, x, v, y)=-\infty$.)

Theorem 1. For $w, v, x, y \in W, k>\ell(w, x, v, y)$ implies

$$
\operatorname{Ext}_{\mathcal{O}}^{k}\left(T_{w} M(x), T_{v} M(y)\right)=0 .
$$

Received by the editors November 14, 2008, and, in revised form, March 2, 2009.

2000 Mathematics Subject Classification. Primary 22E47; Secondary 17B55.

Key words and phrases. Category $\mathcal{O}$, twisted Verma modules.

(C)2009 American Mathematical Society 
Remark 2. If $w=e$, then $w A_{w^{-1}}(x)=x$. Hence $\ell(w, x, v, y)=\ell(x)-\ell(v y)$ (or $-\infty)$. As a special case, this theorem implies that $\operatorname{Ext}_{\mathcal{O}}^{k}(M(x), M(y))=0$ for all $k>\ell(x)-\ell(y)$. This is a result of Schmid [Sch81] and Delorme [Del80]. Moreover,

Carlin [Car86, Theorem 3.8] proved that $\operatorname{dim} \operatorname{Ext}_{\mathcal{O}}^{\ell(x)-\ell(y)}(M(x), M(y))=1$ if $x \geq y$.

\section{Proof of the main theorem}

In the rest of this paper, we denote $\operatorname{Ext}_{\mathcal{O}}$ by Ext. We use the following lemmas.

Lemma 3. (1) Let $s$ be a simple reflection and $w, x \in W$. Assume that $w s>w$ and $s x>x$. Then we have $\ell(w s x)>\ell(w x)$.

(2) Let $s$ be a simple reflection and $t$ a reflection such that $s \neq t$. If $s t w<s w$, then $t w<w$.

Proof. (1) Assume that $\ell(w s x)<\ell(w x)$. Let $w=s_{1} \cdots s_{l}, x=s_{l+1} \cdots s_{r}$ be reduced expressions. Put $t=w s w^{-1}$. Then we have $\ell(t w x)<\ell(w x)$. Hence by the strong exchange condition [Hum90, 5.8], for some $k$ we have $t s_{1} \cdots s_{k}=s_{1} \cdots s_{k-1}$. If $k \leq l$, then $w s=t w=s_{1} \cdots s_{k-1} s_{k+1} \cdots s_{l}<w$; this is a contradiction. If $k>l$, then $s x=w^{-1} t w x=s_{l+1} \cdots s_{k-1} s_{k+1} \cdots s_{r}<x$; this is also a contradiction.

(2) Take a positive root $\beta$ such that $t$ is a reflection with respect to $\beta$. Then sts ${ }^{-1}$ is a reflection with respect to $s(\beta)$. Let $\check{\beta}$ be the coroot corresponding to $\beta$. The assumption $s \neq t$ implies that $s(\check{\beta})$ is positive. Since $s t s^{-1}(s w)=s t w<s w$, we have $\langle s(\breve{\beta}), s w \rho\rangle<0$ by Proposition 5.7 of Hum90. Hence $\langle\breve{\beta}, w \rho\rangle<0$. Therefore, we have $t w<w$.

Lemma 4. Let $s$ be a simple reflection such that $s w>w$. Then we have

$$
A_{s w}(x)= \begin{cases}s A_{w}(s x) & (s x>x), \\ s A_{w}(x) \cup s A_{w}(s x) & (s x<x) .\end{cases}
$$

Proof. Fix a reduced expression $s w=s_{1} \cdots s_{l}$ such that $s=s_{1}$. Then $w=$ $s_{2} \cdots s_{l}$ is a reduced expression of $w$. Put $t_{i}=\left(s_{1} \cdots s_{i-1}\right) s_{i}\left(s_{1} \cdots s_{i-1}\right)^{-1}$ and $t_{i}^{\prime}=s^{-1} t_{i} s=\left(s_{2} \cdots s_{i-1}\right) s_{i}\left(s_{2} \cdots s_{i-1}\right)^{-1}$. Notice that $t_{i} \neq t_{j}$ if $i \neq j$.

By the definition, $z \in A_{s w}(x)$ if and only if there exist $1 \leq i_{1}<\cdots<i_{r} \leq l$ such that $z=t_{i_{r}} \cdots t_{i_{1}} x$ and $t_{i_{k}} t_{i_{k-1}} \cdots t_{i_{1}} x<t_{i_{k-1}} \cdots t_{i_{1}} x$ for all $k=1, \ldots, r$. Then $z=s t_{i_{r}}^{\prime} \cdots t_{i_{1}}^{\prime} s x$ and $s t_{i_{k}}^{\prime} t_{i_{k-1}}^{\prime} \cdots t_{i_{1}}^{\prime} s x<s t_{i_{k-1}}^{\prime} \cdots t_{i_{1}}^{\prime} s x$ for all $k=1, \ldots, r$. If $i_{k} \neq$ 1 , then $s \neq t_{i_{k}}^{\prime}$. Hence we have $t_{i_{k}}^{\prime} t_{i_{k-1}}^{\prime} \cdots t_{i_{1}}^{\prime} s x<t_{i_{k-1}}^{\prime} \cdots t_{i_{1}}^{\prime} s x$ by Lemma 3 (2). If $i_{1} \neq 1$, then we have $s z \in A_{w}(s x)$. If $i_{1}=1$, then $x$ must satisfy $s x=t_{i_{1}} x<x$ and $s z \in A_{w}(x)$. Therefore we get

$$
A_{s w}(x) \subset \begin{cases}s A_{w}(s x) & (s x>x), \\ s A_{w}(x) \cup s A_{w}(s x) & (s x<x) .\end{cases}
$$

The reverse inclusion follows from the above argument.

Lemma 5. If $v s>v$, then we have

$$
\ell(w, x, v, y) \begin{cases}=\ell(w, x, v s, s y) & (s y<y), \\ \geq \max \{\ell(w, x, v s, s y), \ell(w, x, v s, y)+1\} & (s y>y) .\end{cases}
$$

Proof. Fix $x, w \in W$. For simplicity, put $A(v, y)=w A_{w^{-1}}(x) \cap v w_{0} A_{w_{0} v^{-1}}\left(w_{0} y\right)$. First assume that $s y<y$. Put $s^{\prime}=w_{0} s w_{0}$. Then $s^{\prime}$ is also a simple reflection. From 
$s y<y$, we have $s^{\prime} w_{0} y>w_{0} y$. By Lemma 4, $A_{w_{0} v^{-1}}\left(w_{0} y\right)=s^{\prime} A_{s^{\prime} w_{0} v^{-1}}\left(s^{\prime} w_{0} y\right)=$ $s^{\prime} A_{w_{0}(v s)^{-1}}\left(w_{0} s y\right)$. Therefore $A(v, y)=A(v s, s y)$. Hence the definition of $\ell$ implies that $\ell(w, x, v s, s y)=\ell(w, x, v, y)$. Next, assume that $s y>y$. Then using the above argument, we have $A(v, y) \supset A(v s, s y)$. Hence we have $\ell(w, x, v, y) \geq \ell(w, x, v s, s y)$. By Lemma 4, we also have $A(v, y) \supset A(v s, y)$ since $s^{\prime} w_{0} v^{-1}<w_{0} v^{-1}$. Hence $\ell(w, x, v, y) \geq \max \{2 \ell(z)-\ell(w x)-\ell(v y) \mid z \in A(v s, y)\}$. By Lemma 3 (1), we have $\ell(v y)<\ell(v s y)$. Hence we have $\ell(w, x, v, y) \geq \ell(w, x, v s, y)+1$.

Lemma 6. Fix $w, x \in W$. If $\operatorname{Ext}^{k}\left(T_{w} M(x), T_{w_{0}} M(y)\right)=0$ for all $y \in W$ and $k>\ell\left(w, x, w_{0}, y\right)$, then $\operatorname{Ext}^{k}\left(T_{w} M(x), T_{v} M(y)\right)=0$ for all $v, y \in W$ and $k>$ $\ell(w, x, v, y)$.

Proof. We prove by downward induction on $\ell(v)$. The base case of $v=w_{0}$ is just our assumption.

Now assume that $v \neq w_{0}$. Take a simple reflection $s$ such that $v s>v$. Assume first that $s y<y$. Then we have $T_{v} M(y) \simeq T_{v s} M(s y)$ [AL03, Proposition 6.3]. Hence $\operatorname{Ext}_{\mathcal{O}}^{k}\left(T_{w} M(x), T_{v} M(y)\right)=0$ for $k>\ell(w, x, v s, s y)=\ell(w, x, v, y)$.

Next, assume that $s y>y$. In this case, by [AL03, Proposition 6.3], we have the following exact sequence:

$$
0 \rightarrow T_{v s} M(y) \rightarrow T_{v} M(y) \rightarrow T_{v s} M(s y) \rightarrow T_{v s} M(y) \rightarrow 0 .
$$

Put $L=\operatorname{Ker}\left(T_{v s} M(s y) \rightarrow T_{v s} M(y)\right)$. Then we have an exact sequence $0 \rightarrow L \rightarrow$ $T_{v s} M(s y) \rightarrow T_{v s} M(y) \rightarrow 0$. By the corresponding long exact sequence, we have an exact sequence

$$
\operatorname{Ext}^{k-1}\left(T_{w} M(x), T_{v s} M(y)\right) \rightarrow \operatorname{Ext}^{k}\left(T_{w} M(x), L\right) \rightarrow \operatorname{Ext}^{k}\left(T_{w} M(x), T_{v s} M(s y)\right) .
$$

If $k>\ell(w, x, v, y)$, then by Lemma 5 we have $k-1>\ell(w, x, v s, y)$ and $k>$ $\ell(w, x, v s, s y)$. By the inductive hypothesis, we have $\operatorname{Ext}^{k-1}\left(T_{w} M(x), T_{v s} M(y)\right)=$ $\operatorname{Ext}^{k}\left(T_{w} M(x), T_{v s} M(s y)\right)=0$. Hence we get $\operatorname{Ext}^{k}\left(T_{w} M(x), L\right)=0$. We also have an exact sequence $0 \rightarrow T_{v s} M(y) \rightarrow T_{v} M(y) \rightarrow L \rightarrow 0$. From the above argument, we have $\operatorname{Ext}^{k}\left(T_{w} M(x), T_{v s} M(y)\right)=\operatorname{Ext}^{k}\left(T_{w} M(x), L\right)=0$. Therefore we get $\operatorname{Ext}^{k}\left(T_{w} M(x), T_{v} M(y)\right)=0$ from the long exact sequence.

Let $D$ be a dualizing functor of $\mathcal{O}$ (see, for instance, Hum08, 3.2]).

Proof of Theorem 1. It suffices to prove that $\operatorname{Ext}^{k}\left(T_{w} M(x), T_{w_{0}} M(y)\right)=0$ for all $k>\ell\left(w, y, w_{0}, x\right)$ by Lemma 6. First we prove this for $w=e$. By [AL03, Remark 5.1], $T_{w_{0}} M(y) \simeq D M\left(w_{0} y\right)$. Hence $\operatorname{Ext}^{k}\left(M(x), T_{w_{0}} M(y)\right)=0$ if $k>0$ or $x \neq w_{0} y$ by [Hum08, Theorem 6.12 , Theorem 3.3(c)], so we have proved the theorem in this case. (Note: $\ell\left(e, x, w_{0}, y\right)=-\infty$ if $x \neq w_{0} y$.)

By Lemma 6. $\operatorname{Ext}^{k}\left(M(x), T_{v} M(y)\right)=0$ for all $v, x, y \in W$ and $k>\ell(e, x, v, y)$. From [AL03, Remark 5.1], we have $D T_{v} M(y)=T_{v w_{0}} M\left(w_{0} y\right)$. Hence applying $D$, we have $\operatorname{Ext}^{k}\left(T_{v w_{0}} M\left(w_{0} y\right), T_{w_{0}} M\left(w_{0} x\right)\right)=0$ for all $k>\ell(e, x, v, y)$. By definition, we have $\ell(e, x, v, y)=\ell\left(v w_{0}, w_{0} y, w_{0}, w_{0} x\right)$. Consequently, we have $\operatorname{Ext}^{k}\left(T_{v} M(y), T_{w_{0}} M(x)\right)=0$ for all $k>\ell\left(v, y, w_{0}, x\right)$, from which the theorem follows.

\section{ACKNOWLEDGMENTS}

The author is supported by a Japan Society for the Promotion of Science Research Fellowship for Young Scientists. 


\section{REFERENCES}

[Abe] Noriyuki Abe, On the existence of homomorphisms between principal series of complex semisimple Lie groups, preprint.

[AL03] H. H. Andersen and N. Lauritzen, Twisted Verma modules, Studies in memory of Issai Schur (Chevaleret/Rehovot, 2000), Progr. Math., vol. 210, Birkhäuser Boston, Boston, MA, 2003, pp. 1-26. MR1985191 (2004d:17005)

[BG80] J. N. Bernstein and S. I. Gel'fand, Tensor products of finite- and infinite-dimensional representations of semisimple Lie algebras, Compositio Math. 41 (1980), no. 2, 245-285. MR581584 (82c:17003)

[BGG76] I. N. Bernšteřn, I. M. Gel'fand, and S. I. Gel'fand, A certain category of $\mathfrak{g}$-modules, Funkcional. Anal. i Priložen. 10 (1976), no. 2, 1-8. MR0407097 (53:10880)

[Car86] Kevin J. Carlin, Extensions of Verma modules, Trans. Amer. Math. Soc. 294 (1986), no. 1, 29-43. MR819933 (87j:17006)

[Del80] M. Delorme, Extensions in the Bernště̌n-Gel'fand-Gel'fand category $\mathcal{O}$. Applications, Funktsional. Anal. i Prilozhen. 14 (1980), no. 3, 77-78. MR583808 (82j:17009)

[Hum90] James E. Humphreys, Reflection groups and Coxeter groups, Cambridge Studies in Advanced Mathematics, vol. 29, Cambridge University Press, Cambridge, 1990. MR1066460 (92h:20002)

[Hum08] James E. Humphreys, Representations of semisimple Lie algebras in the BGG category $\mathscr{O}$, Graduate Studies in Mathematics, vol. 94, American Mathematical Society, Providence, RI, 2008. MR2428237

[Irv93] Ronald Irving, Shuffled Verma modules and principal series modules over complex semisimple Lie algebras, J. London Math. Soc. (2) 48 (1993), no. 2, 263-277. MR.1231714 (94i:17013)

[Sch81] Wilfried Schmid, Vanishing theorems for Lie algebra cohomology and the cohomology of discrete subgroups of semisimple Lie groups, Adv. in Math. 41 (1981), no. 1, 78-113. MR625335(82h:17009)

Graduate School of Mathematical Sciences, The University of Tokyo, 3-8-1 Komaba, MEguro-Ku, TOKYO 153-8914, JAPAN

E-mail address: abenori@ms.u-tokyo.ac.jp 